\title{
Circadian regulation of intracellular G-protein signalling mediates intercellular synchrony and rhythmicity in the suprachiasmatic nucleus
}

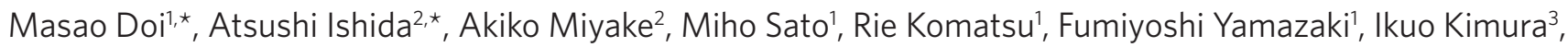 \\ Soken Tsuchiya ${ }^{3}$, Hiroshi Kori, ${ }^{4,5}$ Kazuyuki Seo', Yoshiaki Yamaguchi', Masahiro Matsuo', Jean-Michel Fustin', \\ Rina Tanaka', Yasuko Santo', Hiroyuki Yamada', Yukari Takahashi', Michihiro Araki ${ }^{6}, K^{1}$ Kazuki Nakao ${ }^{7}$, \\ Shinichi Aizawa7, Masaki Kobayashi ${ }^{8}$, Karl Obrietan, Gozoh Tsujimoto ${ }^{3}$ \& Hitoshi Okamura, ${ }^{1,2}$
}

Synchronous oscillations of thousands of cellular clocks in the suprachiasmatic nucleus (SCN), the circadian centre, are coordinated by precisely timed cell-cell communication, the principle of which is largely unknown. Here we show that the amount of RGS16 (regulator of G protein signalling 16), a protein known to inactivate Goi, increases at a selective circadian time to allow time-dependent activation of intracellular cyclic AMP signalling in the SCN. Gene ablation of Rgs16 leads to the loss of circadian production of CAMP and as a result lengthens circadian period of behavioural rhythm. The temporally precise regulation of the cAMP signal by clockcontrolled RGS16 is needed for the dorsomedial SCN to maintain a normal phase-relationship to the ventrolateral SCN. Thus, RGS16-dependent temporal regulation of intracellular G protein signalling coordinates the intercellular synchrony of SCN pacemaker neurons and thereby defines the $24 \mathrm{~h}$ rhythm in behaviour.

\footnotetext{
${ }^{1}$ Department of Systems Biology, Graduate School of Pharmaceutical Sciences, Kyoto University, Kyoto 606-8501, Japan. ${ }^{2}$ Division of Molecular Brain Science, Graduate School of Medicine, Kobe University, Kobe 650-0017, Japan. ${ }^{3}$ Department of Genomic Drug Discovery Science, Graduate School of Pharmaceutical Sciences, Kyoto University, Kyoto 606-8501, Japan. ${ }^{4}$ Division of Advanced Sciences, Ochadai Academic Production, Ochanomizu University, Tokyo 112-8610, Japan. ${ }^{5}$ PRESTO, Japan Science and Technology Agency, Kawaguchi 332-0012, Japan. ${ }^{6}$ Education Unit for Global Leaders, Kyoto University, Kyoto 606-8501, Japan. ${ }^{7}$ Laboratory for Animal Resources and Genetic Engineering, Riken Center for Developmental Biology, Kobe 650-0047, Japan. ${ }^{8}$ Department of Electronics, Tohoku Institute of Technology, Sendai 982-8577, Japan. ${ }^{9}$ Department of Neuroscience, The Ohio State University, Ohio 43210, USA. *These authors contributed equally to this work. Correspondence and requests for materials should be addressed to H.O. (email: okamurah@pharm.kyoto-u.ac.jp).
} 
n mammals, the hypothalamic suprachiasmatic nucleus (SCN) is a central pacemaker, governing daily rhythms in behaviour and physiology ${ }^{1-3}$. Individual neurons in the SCN act as cell-autonomous oscillators, exhibiting circadian fluctuations of firing rate and gene expression ${ }^{4-7}$. We and others have recently shown that autonomous SCN neuronal oscillators maintain a high degree of synchrony with each other, so that they can generate coherent signals required for the circadian control of behaviour and physiology ${ }^{6-10}$.

While individual SCN neurons share the same circadian period as a result of synchronization, the phases of their rhythms are strikingly diverse ${ }^{6}$. This observation reflects the reality that the SCN is an anatomically heterologous cellular assemblage, in which cohorts of differentially phased cellular clocks are deployed in a topographically specific manner ${ }^{11,12}$ : neurons in the dorsomedial part of the SCN reach their daily peak in clock gene expression earlier in the day than those in the rest of the SCN. However, the mechanism by which dorsomedial SCN cells maintain their relatively advanced phasing, and to what extent this differential phasing influences the rhythms in behaviour have remained unknown.

Much better understood is the molecular mechanism underlying cell-autonomous circadian oscillations. These rely on the opposing effects of transcriptional activators and repressors that generate negative feedback loops ${ }^{13-15}$. Briefly, the transcriptional activators CLOCK and BMAL1 drive transcription of Period (Per1 and Per2) and Cryptochrome (Cry1 and Cry2) genes via binding to E-box elements present in their promoters. Once the repressor proteins PER and CRY reach a critical concentration, they form complexes and repress their own expression by inhibiting the CLOCK/BMAL1 complexes. In addition to E-box elements, the promoter regions of Per genes contain D-boxes, through which the PAR bZip family of transcriptional activators (DBP, TEF and HLF) and the related repressor E4BP4 exert their opposing effects on the expression of target genes ${ }^{16-20}$. Finely controlled transcriptional regulation through the E-box and D-box motifs is thought to generate robust circadian expression of Per genes as well as a wide variety of clock-controlled genes ${ }^{21}$.

Although cell-autonomous transcriptional regulation of clock genes could provide a common mechanism underlying the oscillations of individual cells, this does not explain the phasic differences between the neurons in the SCN. In this regard, it is important to note that the promoter region of Per1 gene contains a functional cyclic AMP-responsive element ${ }^{22}$, and that the extracellular signalling that activates cAMP pathway can acutely induce Per1 expression $^{23}$. Because a light pulse given in the night time induces Per1 expression in the SCN, this pathway has long been considered crucial for light-dependent resetting of the phase of the molecular clock $^{24-26}$. Yet, the cAMP-dependent control of Per1 may also have a role in coordinating circadian rhythms (phase-arrangement) of neurons in the $\mathrm{SCN}^{14}$. Supporting this hypothesis, the concentration of CAMP and transcriptional activity of the cAMP-responsive element in the SCN are both highly rhythmic even in constant darkness $^{27-29}$. Moreover, pharmacological reduction in the rate of cAMP synthesis in the SCN lengthens the circadian period of the behavioural rhythm ${ }^{29}$, demonstrating a role of cAMP in the determination of the circadian period.

RGS16 is a potential regulator of rhythmic cAMP synthesis in the SCN. Biochemically, RGS16 functions as a GTPase-accelerating protein for $\mathrm{G} \alpha \mathrm{i} / \mathrm{o}$ subtypes of $\mathrm{G}$ protein $\alpha$ subunits $^{30-32}$. By promoting GTP hydrolysis, RGS16 terminates GTP-bound (thus activated) Goi/o signalling. Microarray studies and the subsequent expression analyses revealed that the gene encoding RGS16 exhibits circadian variations in the levels of transcript in the $\mathrm{SCN}^{33-36}$. These data raise the possibility that Goi/o-mediated cAMP signalling in the SCN is under circadian regulation via RGS16.

Here we show that gene ablation of Rgs16 leads to the loss of circadian production of CAMP in the SCN, which results in a period lengthening of the locomotor activity rhythm. Extensive analysis of the intercellular phase-relationship within the SCN reveals that the RGS16-mediated daily activation of cAMP is necessary to accelerate the expression of Per1 in the dorsomedial cells. Our data therefore demonstrate that RGS16-mediated cAMP signalling defines the advanced phasing of dorsomedial cells, and as such, is a critical determinant for the period of circadian rhythms in behaviour.

\section{Results}

Gene expression profiles of the RGS family in the SCN. It is known that pharmacological inhibition of Goi/o activity with pertussis toxin hampers intercellular synchronization ${ }^{37}$ and causes dampened rhythms of the entire SCN (Supplementary Fig. S1) ${ }^{37}$. These observations prompted us to hypothesize that member(s) of the regulator of G protein signalling (RGS) family might contribute to synchronized cellular oscillations in the SCN. We surveyed all known mouse Rgs genes for their expression in the SCN by using DNA microarray analysis (Supplementary Fig. S2a). Then, the genes highly expressed in the SCN were selected and further analysed with quantitative in situ hybridization to specify their histological distribution and circadian time (CT)-dependent expression profile (Supplementary Fig. S2b) ${ }^{38}$. This survey led us to notice that $R g s 16$ is the only RGS family member that displays marked SCN-specific expression particularly in the subjective daytime (see CT4 in Fig. 1a; CT4 corresponds to $4 \mathrm{~h}$ after subjective onset of daytime under constant dark conditions). This expression profile, together with a prior work showing that RGS16 regulates Goi/o activity ${ }^{31,32}$, raised the prospect that RGS16 contributes to SCN circadian clock physiology.

Cyclic expression of Rgs16 is driven by the circadian clock. Timedependent profiling revealed that Rgs16 exhibits overt circadian expression in the SCN (Fig. 1b), which persists even in constant darkness (DD) with a drastic increase of expression in the early subjective morning. Topographical analysis by digoxigenin in situ hybridization (Fig. 1c) further demonstrated that Rgs16 expression began to increase by subjective dawn (CT0) in the periventricular part of the dorsomedial SCN (a narrow but cell-dense area near the third ventricle). Thereafter, Rgs16-positive staining spread widely across the SCN at CT4. At CT8, Rgs16 levels began to decrease in all SCN regions, and throughout the subjective night (that is, CT1220) expression was limited to a few SCN cells. The spatiotemporal expression of Rgs16 resembles that of Per1, a clock gene showing peak messenger RNA expression during the daytime in the $\mathrm{SCN}^{11,12}$. Similar to the Per 1 promoter, the $5^{\prime}$-flanking genomic sequence of Rgs16 contains E-box and D-box cis-regulatory elements, thus allowing the circadian regulators CLOCK-BMAL1 and DBP to activate transcription. In a further parallel with Per1, CRY1 and E4BP4 repressed CLOCK-BMAL1- and DBP-evoked Rgs16 expression, respectively (Supplementary Figs. S3 and S4). Consistent with the promoter characteristics, in vivo circadian expression of Rgs16 was dramatically altered in the PAR bZip proteins-deficient mice

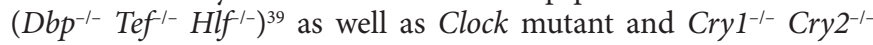
mice (Supplementary Fig. S4f). In Clock mutant mice, the early subjective day peak in Rgs16 expression was damped whereas in Cry $1^{-/-} \mathrm{Cry} 2^{-/-}$mice Rgs16 expression was high at both the subjective day and night time points. These data clearly demonstrate that expression of Rgs16 is under the control of the intracellular clock machinery. It is also noteworthy that the expression of $\mathrm{Rgs} 16$ was not light-inducible (Supplementary Fig. S5). Thus, the internal clock seems to drive the morning expression of Rgs16 in the SCN.

RGS16 deficiency lengthens the circadian period of behaviour. To characterize the in vivo function of RGS16, we generated RGS16deficient mice, in which a genomic region spanning whole coding sequence of $R g s 16$ was deleted and replaced with a lacZ reporter cassette (Fig. 1d,e). In situ hybridization analysis (Fig. 1f) con- 
a

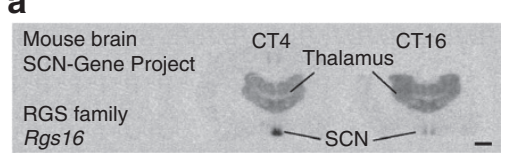

b

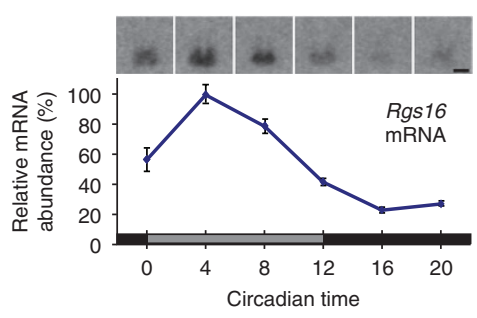

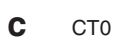

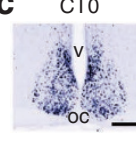

CT4

Ст8

CT12

CT16

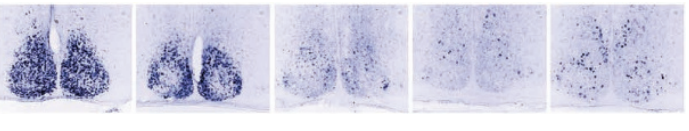

CT20

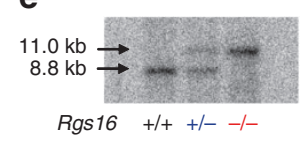

d
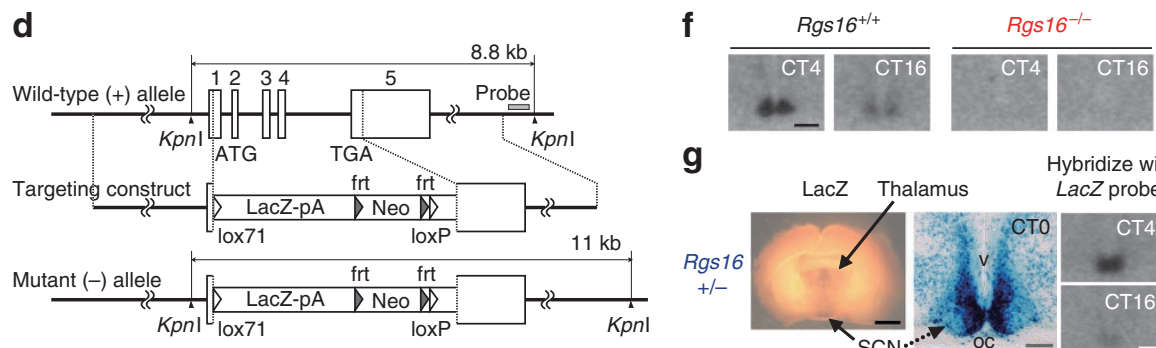

g

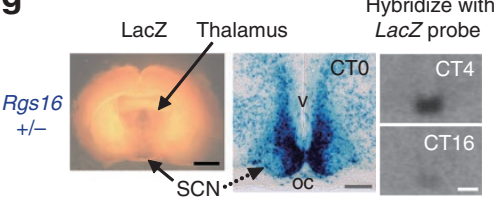

h

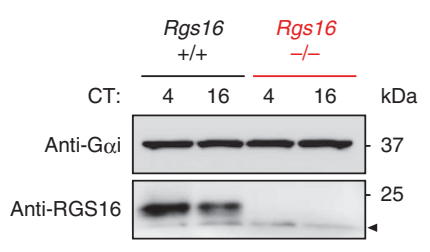

i

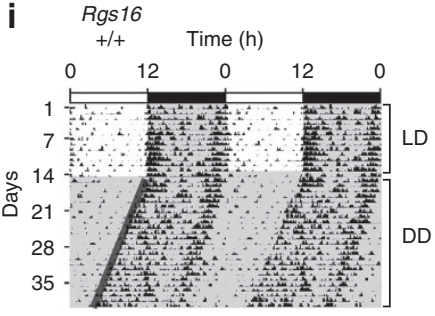

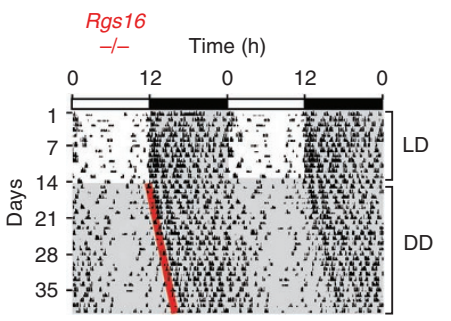

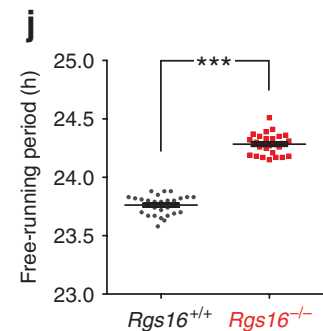

Figure 1 | RGS16 deficiency lengthens the circadian period of the locomotor activity rhythm. (a) Autoradiographs showing mouse coronal brain sections hybridized with an Rgs16 probe. CT4 and CT16 correspond respectively to 4 and $16 \mathrm{~h}$ after the subjective onset of daytime in DD. Bar, $1 \mathrm{~mm}$. (b) Circadian expression of Rgs16 in the SCN in DD. Relative RNA abundance was quantified with radioisotopic in situ hybridization. Values means \pm s.e.m ( $n=5)$ with the peak value adjusted to 100. Representative autoradiographs at the indicated time points are shown on the top. Bar, $200 \mu \mathrm{m}$. (c) Digoxigenin in situ hybridization showing the distribution of Rgs16-positive cells in the SCN. oc, optic chiasm; v, third ventricle. Bar, $100 \mu$ m. (d) Schematic representation of the mouse Rgs16 gene, targeting construct, and the mutant allele. Numbered boxes are the exons. The DNA probe used for southern blots is marked by a grey box. (e) Southern blot of Kpnl-digested DNA from Rgs16 ${ }^{+/+}$, Rgs $16^{+/-}$and Rgs $16^{-1-}$ mice, WT (8.8 kb) and mutant (11 kb) allele. (f) Radioisotopic in situ hybridization autoradiographs showing WT and Rgs16 $6^{-/}$SCN. Bar, $200 \mu \mathrm{m}$. (g) Rgs $16^{+/-}$coronal brain slice stained with X-gal (blue) with a magnified view of the SCN in a thin brain section at CTO. Autoradiographs show quantitative LacZ mRNA in situ hybridization at CT4 and CT16. Scale bars: black, $1 \mathrm{~mm}$, grey, $100 \mu \mathrm{m}$, white, $300 \mu \mathrm{m}$. (h) Western blots of RGS16 and Goi in the SCN at CT4 and CT16. Arrowhead indicates a non-specific band. (i) Representative locomotor activity records of C57BL/6J-backcrossed Rgs16 ${ }^{+/+}$and Rgs16 $/ /-$mice. Mice were housed in a 12L:12D light-dark cycle and then transferred to DD. Periods of darkness are indicated by grey backgrounds. The data are shown in double-plotted format. Each horizontal line represents $48 \mathrm{~h}$; the second 24 -h period is plotted to the right and below the first. The coloured lines delineate the phase of activity onset in DD. (j) Period-length distribution of C57BL/6J-backcrossed $\mathrm{Rgs} 16^{+/+}$and $\mathrm{Rgs} 16^{-/-}$mice. Free-running period measurements were based on a 14-day interval taken after 3 days of a DD regime and were executed with a $\chi^{2}$ periodogram. Plotted are the period lengths of individual animals. Bars indicate mean \pm s.e.m. ${ }^{\star \star \star} P<0.001$, Student's $t$-test.

firmed the loss of the Rgs16 transcript in Rgs16 homozygous mutant $\left(\right.$ Rgs $\left.16^{-1-}\right)$ mice. $\beta$-galactosidase tissue staining (Fig. $\left.1 \mathrm{~g}\right)$ further corroborated gene targeting and replacement by LacZ: the spatio-temporal expression of the transgene reflected Rgs16 expression in the SCN. Moreover, we generated an antibody to RGS16 and performed immunoblot analysis with SCN extracts (Fig. 1h). As expected, the expression of RGS16 was not detected in $\mathrm{Rgs}_{16}{ }^{-1-}$ mice, whereas wild-type (WT) mice exhibited an increased expression of RGS16 protein in the subjective morning (Fig. 1h). We thus conclude that Rgs16 mutant mice are indeed RGS16-deficient.

Behavioural analysis was performed with Rgs16 mutant mice that had been backcrossed to the C57BL/6J background over ten generations. We observed that the gene ablation of $\mathrm{Rgs} 16$ causes a lengthening of circadian period of the locomotor activity rhythm (Fig. 1i): under constant darkness, all $R g s 16^{-1-}$ mice tested $(n=27)$ showed a free-running period longer than $24 \mathrm{~h}$ (circadian period (mean \pm s.e.m) determined with a $\chi^{2}$ periodogram, $24.28 \pm 0.01 \mathrm{~h}$ ). The phenotype of Rgs16 knockout sharply contrasts with that of Rgs $16^{+/+}$control mice $(n=29)$, whose period is shorter than $24 \mathrm{~h}$ $(23.76 \pm 0.01 \mathrm{~h})$. The difference between the two genotypes is statistically significant (Fig. 1 j, $P<0.001$, Student's $t$-test) and does not depend on the way of measuring circadian period (periods deter- mined with a linear regression line method: $\operatorname{Rgs}^{+/+}, 23.77 \pm 0.01$ h; $R g s 16^{-1-}, 24.31 \pm 0.01 \mathrm{~h}, P<0.001$, Student's $t$-test), revealing that Rgs16 is a critical determinant of the behavioural rhythm period length. In contrast to the altered circadian periodicity under constant darkness, $\mathrm{Rgs} 16^{-1-}$ mice displayed a normal $24 \mathrm{~h}$ rhythm when maintained in 12-h-light/12-h-dark cycle (Fig. 1i) and their resetting response to early and late night light pulses were indistinguishable from WT mice (Supplementary Fig. S6). Thus, RGS16 is not necessary for light entrainment of the circadian clock. Rather, signalling through RGS16 comprises a pathway responsible for the maintenance of the normal circadian rhythm.

RGS16 targets G $\alpha$ i and regulates cAMP production in the SCN. RGS16 is known to negatively regulate Goi proteins, which downregulate cAMP production by inhibiting adenylate cyclase (AC) activity $^{31,32}$. Double in situ hybridization revealed that both Gnail mRNA and Gnai2 mRNA (encoding Goil and Goi2, respectively) colocalize with Rgs16 in SCN neurons (Fig. 2a). Intriguingly, whereas the levels of Goil and Goi2 proteins were nearly constant throughout the day, the amount of RGS16 protein in the SCN showed overt circadian oscillation (Fig. 2b). Consistent with the GTPase-accelerating protein activity of RGS16, protein pull- 
a

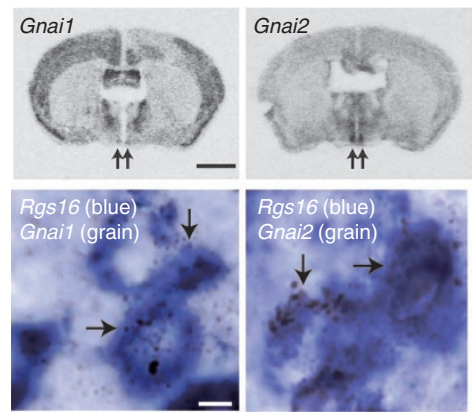

b

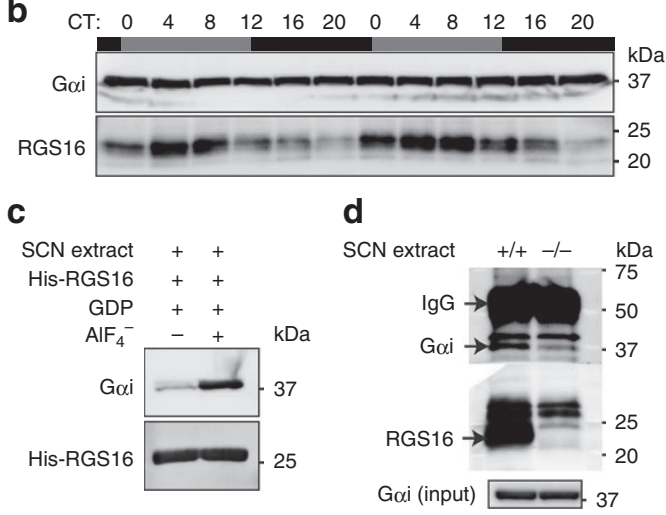

e

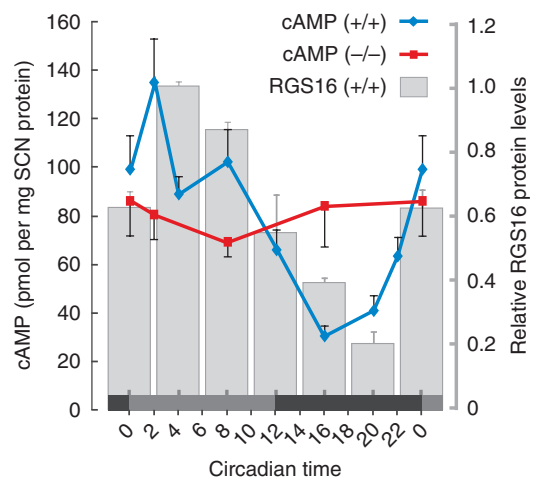

Figure 2 | RGS16 protein targets Goi and regulates circadian production of cAMP in the SCN. (a) (Upper) Low-power film autoradiography showing the mouse coronal brain sections in situ hybridized with radioisotopic probe for Gnail or Gnai2. Arrows indicate the positions of the SCN. Bar, 2 mm. (Lower) High-power photomicrographs of the SCN double in situ hybridized with digoxigenin-labelled probe for Rgs16 (blue) and isotope-labelled probe for Gnail or Gnai2 (silver grains). Arrows indicate representative double-labelled cells. Bar, $5 \mu \mathrm{m}$. (b) Western blots analysing the temporal changes in the protein levels of RGS16 and Goi in the WT mouse SCN. The animals kept in DD were killed at 4-h intervals across two circadian cycles (sampling times indicated at the

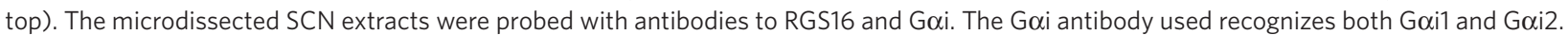

(c) Increased binding affinity of RGS16 and Goi in aluminium fluoride-treated SCN extracts. The microdissected SCN extract was incubated with Histagged RGS16 protein in the presence of GDP with or without aluminium fluoride. Shown are Western blots of the proteins affinity purified with Ni-NTA beads. (d) Immunoprecipitation (IP) of endogenous Goi with endogenous RGS16. The microdissected SCN extracts from Rgs16 ${ }^{+/+}$or Rgs16 ${ }^{-/-}$mice at CTO (input) were subjected to IP with RGS16 antibody. Shown are Western blots of the immunoprecipited RGS16 and Goi (upper) and input Goi (lower). (e) Circadian changes of cAMP content in the microdissected SCN from Rgs16 ${ }^{+/+}$(blue) and Rgs $16^{-/-}$(red) mice (means \pm s.e.m, $n=10$, for each time point). $P<0.01$, CT2 (peak) versus CT16 (trough) in Rgs $16^{+/+}$mice. Such a temporal difference is not detected in Rgs $16^{-/-}$mice. To characterize more precisely the CAMP rhythm seen in WT mice, CAMP contents in the WT SCN were determined at shorter sampling intervals. The bar graph shows the levels of RGS16 protein in the SCN (means \pm range of variation) assessed from the results in $\mathbf{b}$ by densitometric analysis.

down assays demonstrated that Goi proteins in the SCN displayed an increased binding affinity for RGS16 when SCN extracts were treated with aluminium fluoride $\left(\mathrm{AlF}_{4}{ }^{-}-\mathrm{GDP}\right.$ complex forms a transition state analogue of GTP hydrolysis) (Fig. 2c). Furthermore, immunoprecipitation of endogenous RGS16 protein at CT0 showed that the Goi proteins coprecipitated with RGS16 from the protein extract of the WT SCN, but not from that of the $\mathrm{Rgsl6}^{-1-}$ SCN (Fig. 2d).

Time-of-day specific inhibition of Goi by RGS16 might affect circadian production of CAMP in the SCN, which has recently been proved crucial for the maintenance of robust circadian oscillations ${ }^{29}$. Consistent with this model, we found that cAMP concentrations in the WT SCN fluctuated in a circadian fashion, rising synchronously with the expression of RGS16 in the subjective morning (Fig. 2e). Furthermore, we observed that the circadian fluctuation of cAMP was abolished as a result of RGS16 deletion (Fig. 2e). Interestingly, in $\mathrm{Rgs}_{16} \mathrm{6}^{-1-}$ mice, cAMP was maintained at intermediate levels throughout the day, implying that compensatory processes augment cAMP production. Hence, our data indicate that RGS16 is indispensable for conferring circadian rhythmicity (that is, time-of-day specificity) on the production of CAMP in the SCN.

Delayed Per1 cycling in RGS16-deficient dorsomedial cells. The Perl gene is a central clock component that is linked to cAMP signalling through a cAMP-responsive element within its promoter region $^{22}$ (Supplementary Fig. S3). Therefore, we examined the integrity of Per1 expression in the $\mathrm{Rgs}_{16^{-1-} \mathrm{SCN}}$ (Fig. 3). Although circadian expression of Per1 in the mutant SCN was grossly normal, close inspection with digoxigenin in situ hybridization led us to identify a defect of Per1 expression in a small subset of cells located in the periventricular part of the dorsomedial SCN (Fig. 3a). Intriguingly, this region is anatomically characterized by a cluster of 'phase-leading' cells ${ }^{11,12}$ in which Per1 mRNA levels peak at the beginning of the day, preceding the Per 1 mRNA peak in ventral SCN cells. We observed that in $\operatorname{Rgs}_{16} 6^{-1-} \mathrm{SCN}$, the rising phase of
Per1 mRNA expression in the periventricular part of the dorsomedial SCN is significantly delayed, relative to that of WT SCN (Fig. 3a and detailed profiles of Per1 expression in Supplementary Figs. S7 and S8). Thus, we speculated that cAMP signalling through RSG16 might be crucial for the phase-advanced expression of Per 1 at dawn in the dorsomedially located phase-leading cells.

To complement these observations, we analysed the Per1 expression profile in organotypic brain slices from Per 1 promoterluciferase (Per1-luc) transgenic mice ${ }^{6}$ (Fig. 3b-f). Real-time bioluminescence imaging with high-resolution video microscopy ${ }^{6}$ allows us to monitor the circadian dynamics of hundreds of individual neurons in morphologically preserved SCN slices ${ }^{40}$, where the luminescence driven by the Per 1 promoter recapitulates the topographic- and time-dependent expression of Per1 mRNA (Fig. 3b-f, Supplementary Fig. S9 and Supplementary Movie 1, Per1-luc Rgs $\left.16^{+1+} \mathrm{SCN}\right)$. Thus, the phase-advanced oscillation, observable ex vivo in WT dorsomedial cells (Fig. 3b-f) is a property inherent to the SCN ${ }^{6}$. Taking advantage of this system, we examined Per1-luc rhythms in SCN slices from $\mathrm{Rgs}_{16^{-/}}$mice (Fig. 3b-f, Supplementary Fig. S9 and Supplementary Movie 1, Per1-luc Rgs16 ${ }^{-1-}$ SCN). In line with in vivo data from $\mathrm{Rgs} 16^{-1-}$ mice, the luminescence of the $R g s 16^{-1-}$ SCN slice was basically circadian, and its period was relatively long compared with that of WT slice (circadian period (h), mean \pm s.e.m., $R g s 16^{+/+}, 24.35 \pm 0.03 ; R_{g s 16^{-/}}, 24.82 \pm 0.07, n=6$ for each genotype, $P<0.05, t$-test). Furthermore, when the phase differences across three SCN zones (dorsal, middle and ventral) were examined, the dorsal zone, which includes phase-leading cells, exhibited a delayed (relative to $R g s 16^{+/+}$) circadian oscillation, with a resultant phase close to that of the remaining zones (Fig. $3 \mathrm{~d}$ and Supplementary Fig. S9b). Importantly, such alteration of dorsal rhythm was observed in all $\mathrm{Rgs} 16^{-1-} \mathrm{SCN}$ slice cultures tested, and statistical analysis revealed that the luminescence from the dorsal zone of the $\operatorname{Rgs} 16^{-1-} \mathrm{SCN}$ reached its peak at a time significantly later than that of the $\operatorname{Rgs} 16^{+/+} \mathrm{SCN}$ (Fig. 3e and Supplementary Fig. S9c). 
a

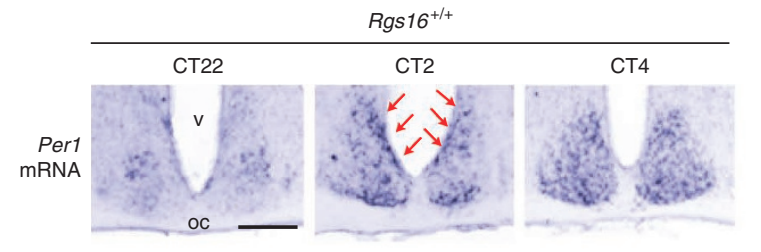

b

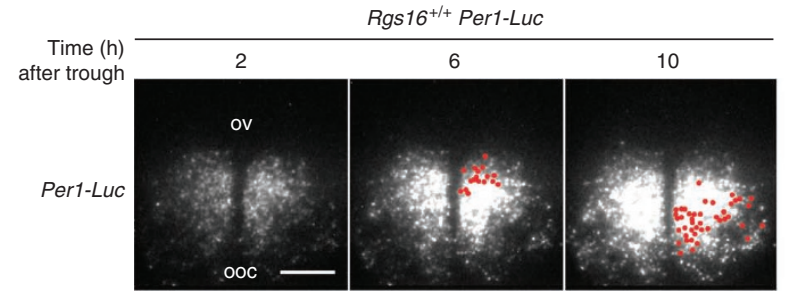

C
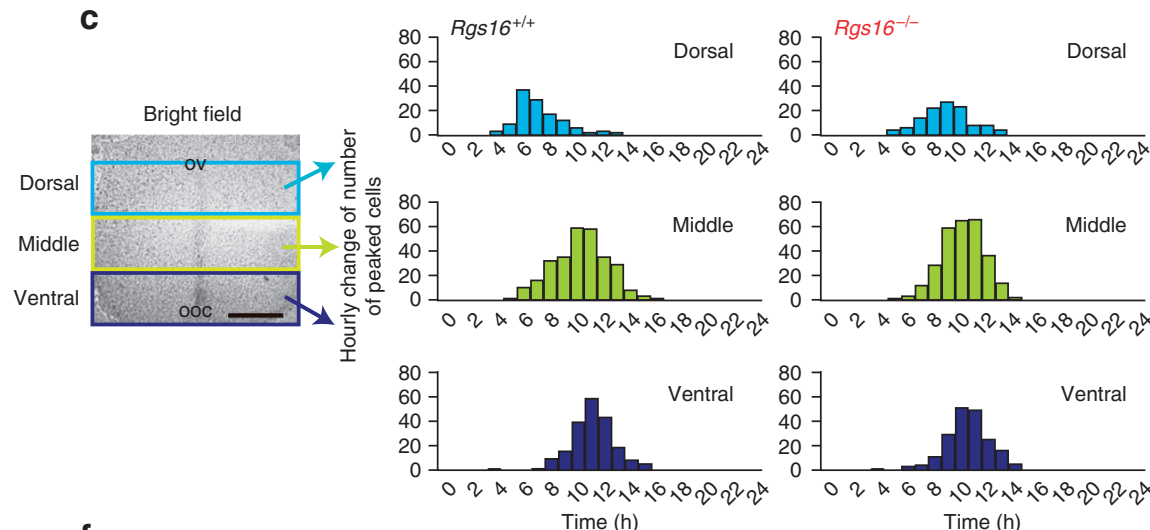
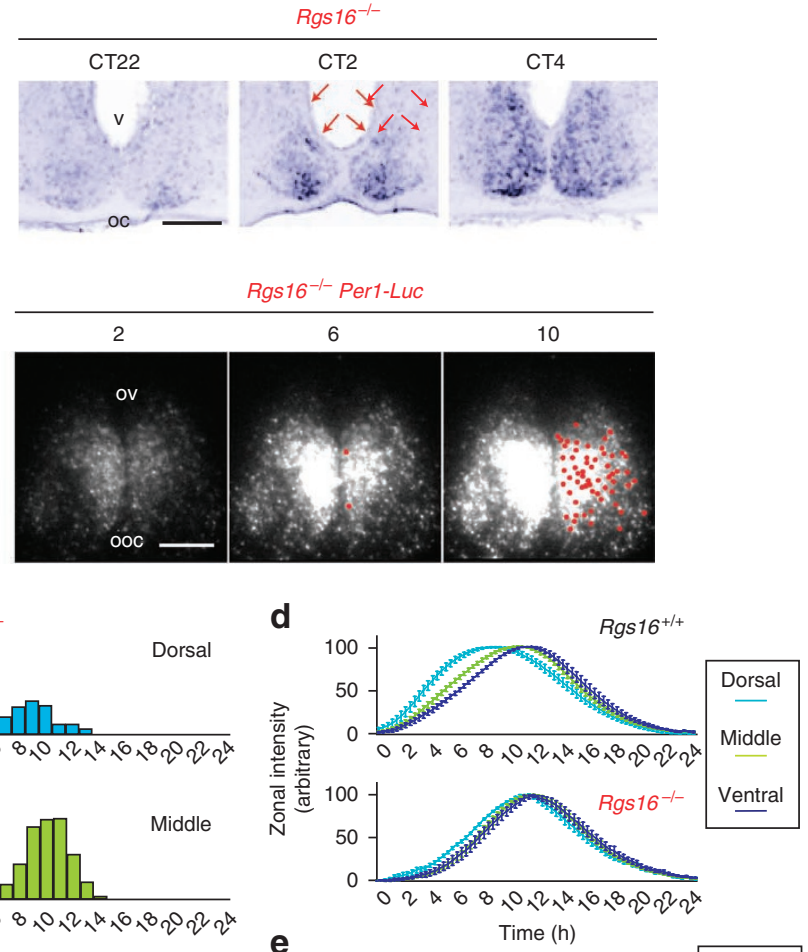

e

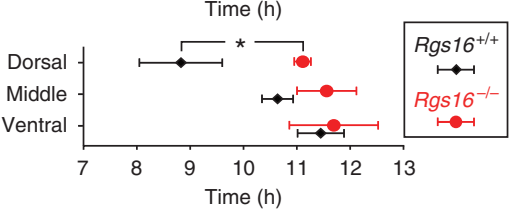

f

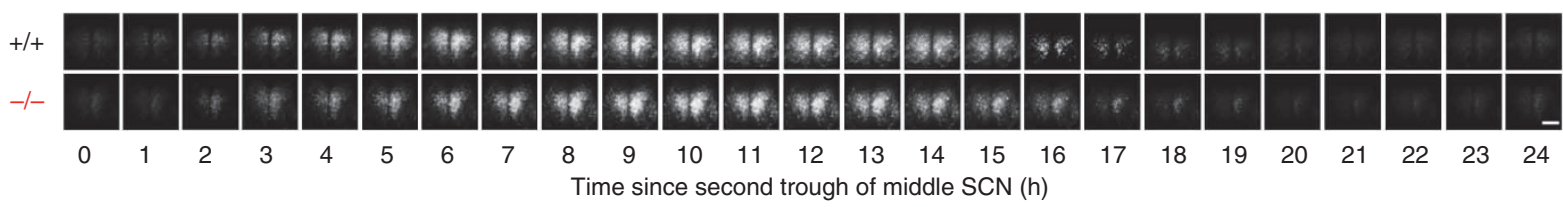

Figure 3 | RGS16 deficiency impairs Per1 expression in dorsomedially located phase-leading cells. (a) Digoxigenin in situ hybridization photomicrographs showing the topographical distributions of Per7-positive cells in the SCN at CT22, CT2 and CT4. (b) Bioluminescent images of whole SCN slices from Per7luc -bearing $\mathrm{Rgs}_{16} \mathrm{H}^{++}$and $\mathrm{Rgs} 16^{-/-}$mice. The red spots on the right-side nucleus indicate the positions of the peaked cells at the indicated time point after the trough of the total SCN bioluminescence. (c) The range of peak times of randomly chosen cells from the subdivided dorsal ( $n=116$, for each genotype), middle $(n=281)$ and ventral $(n=193)$ SCN zones of WT and mutant slices. For non-biased definition of the regions of interest, the SCN was divided into three regions with equal proportions along vertical axis (from the dorsal-most to the ventral-most). The $x$ axis represents time ( $h$ ) since $2 n d$ trough of middle SCN luminescence. Note that the region selected as a dorsal zone encompasses the phase-leading cells. (d) The temporal changes in the levels of total luminescence from each zone (means \pm s.e.m $(n=4)$; the peak and trough values were adjusted to 100 and 0 , respectively). Two-way ANOVA with repeated measures identify a statistically significant interaction between zone and genotype $(F(2,18)=6.88, P<0.001)$. Bonferroni's post hoc tests reveal statistically significant difference between WT and $R g s 16^{-1-}$ slices only in dorsal zone, but not in middle and ventral zones. (e) The average times of peaks ( \pm s.d.) from subdivided dorsal, middle and ventral SCN zones of WT and mutant slices ( $n=4$, for each genotype). ${ }^{\star} P<0.001$ (Bonferroni test). (f) Hourly images of the SCN slices from Rgs16 ${ }^{+/+}$and $R g s 16^{-/-}$mice. See also Supplementary Movie 1. Scale bars in (a-f) are $100 \mu \mathrm{m}$. $v$, third ventricle; ov, original third ventricle; oc, optic chiasm; ooc, original optic chiasm.

Impaired cAMP signalling in RGS16-deficient dorsomedial cells. The observations thus far indicate that dorsomedial SCN cells are the major (or sole) site where the RGS16-cAMP pathway could contribute to the rising phase of the Per1 mRNA. Consistent with this finding, we observed that the dawn expression of Per1 in the dorsomedial cells was unique in that it accompanied a strong induction of cFos (Fig. 4a and Supplementary Fig. S10) ${ }^{41,42}$. as well as a simultaneous activation of extracellular signal-regulated kinase (ERK) (Fig. $4 \mathrm{~b}$ and Supplementary Fig. S10 $)^{43-45}$. Of note, both of these events were cAMP-dependent (Fig. 4c-e); hence, the pharmacological inhibition of cAMP synthesis in vivo with 9-(tetrahydro-2-furyl)adenine (THFA, a noncompetitive AC inhibitor, which slows the rate of cAMP production but does not completely deplete $\mathrm{CAMP}^{29}$ ) suppressed the dawn induction of $c$ Fos and phospho-ERK that otherwise appeared in the periventricular part of the dorsomedial SCN (Fig. 4c-e and Supplementary Fig. S10). Thus, the activated cAMP pathway at dawn is a feature characterizing the phase-leading cells.

Importantly, the dorsomedial cell-specific induction of $c$ Fos and ERK phosphorylation at dawn were both severely compromised by RGS16 deficiency (Fig. 4a,b), demonstrating that RGS16 determines the state of cAMP-dependent signalling in the phase-leading cells. We also observed that the animals treated with THFA phenocopied Rgs $16^{-1-}$ mice (Figs. 1i and 4c), displaying a relatively long circadian locomotor activity rhythm (Figs. $1 \mathrm{j}$ and $4 \mathrm{f}$ ). Thus, we concluded that cAMP signalling at dawn in the phase-leading cells depends on RGS16, and that the loss of RGS16-cAMP signalling causes a 

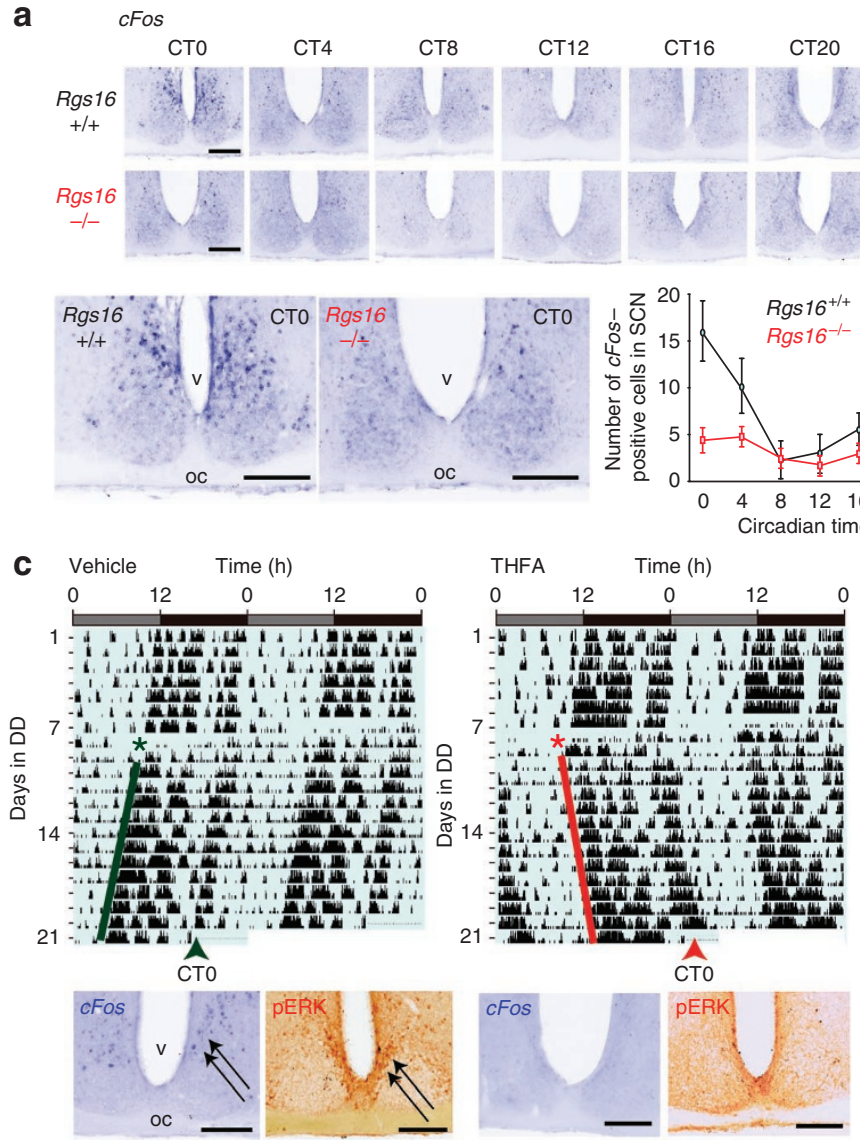

b PERK
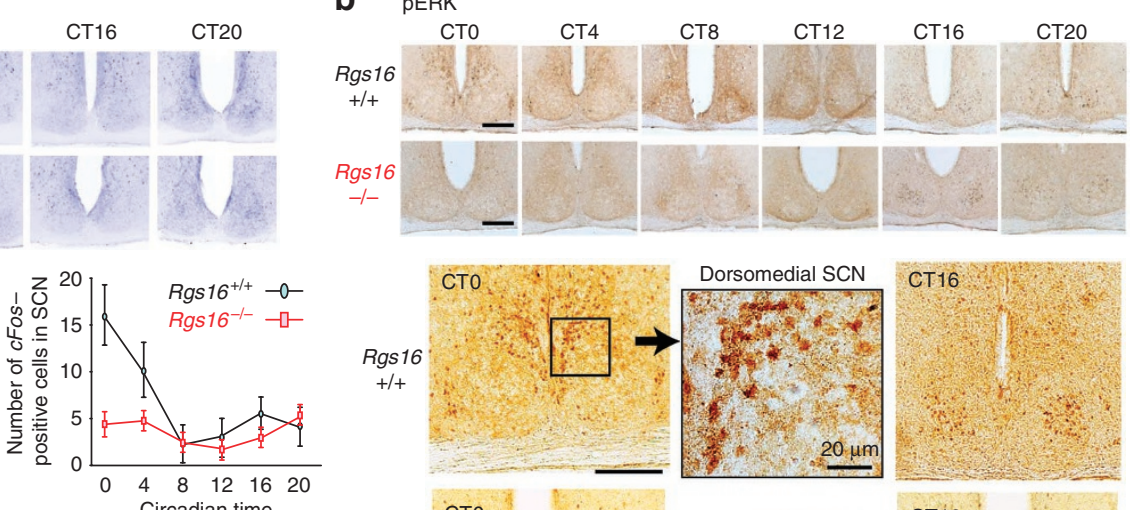
Dorsomedial SCN

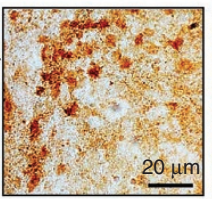
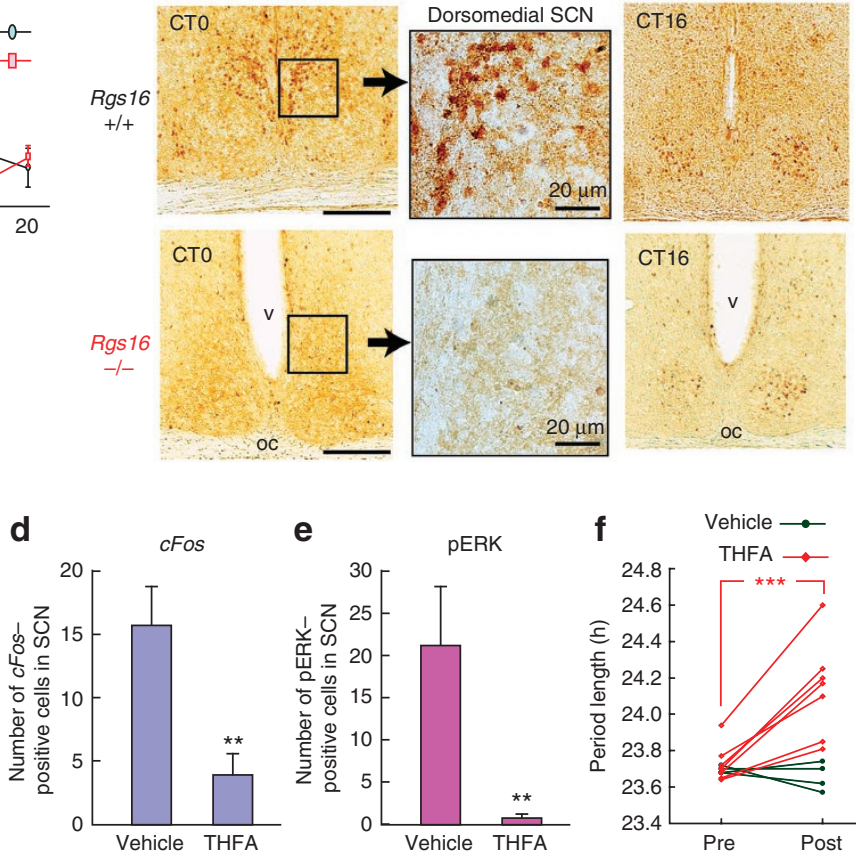

Figure 4 | RGS16 deficiency impairs cAMP-dependent induction of cFos and ERK phosphorylation. (a) Digoxigenin in situ hybridization photomicrographs showing the circadian profiles of cFos expression in the SCN of Rgs $16^{+/+}$and $R g s 16^{-/-}$mice under DD conditions. The graph indicates the number of cFos-positive cells (per section) across the circadian time (means \pm s.e.m, $n=5$ ). (b) Immunoreactivity to the pERK in the SCN of Rgs $16^{+/+}$and $\mathrm{Rgs} 6^{-/-}$mice under DD conditions. (c) Representative locomotor activity records of WT mice under DD conditions before and after the surgery (asterisk) for the continuous infusion of THFA or vehicle (delivered to third ventricle just upper to the SCN via osmotic minipump). Arrowheads indicate the day on which the mice were killed at CTO (estimated by the phase of the activity onset) for the analysis of cFos and pERK expression in the SCN. Representative photomicrographs are shown below the locomotor activity records. Scale bars in a-c are $100 \mu \mathrm{m}$ unless otherwise noted. (d, e) The histograms showing the number (means \pm s.e.m) of cFos- (d) or pERK- (e) positive cells in the SCN (per section) at CT0 after 14-days infusion of either THFA ( $n=6$ ) or vehicle $(n=6)$ in DD. ${ }^{\star \star} P<0.01, t$-test, compared with vehicle treatments. (f) Pair-wised comparisons of behavioural periods before and after infusion of THFA $(n=6)$ or vehicle $(n=4){ }^{\star \star \star} P<0.001$, paired $t$-test in THFA-treated mice. pERK, phosphorylated form of ERK.

lengthening of the circadian period (or a constantly delayed phase) of the locomotor activity rhythm of mice.

\section{Discussion}

A critical issue for understanding SCN function is to identify how individual cellular clocks synchronize with a defined intercellular phase-relationship to broadcast coherent circadian signals to peripheral tissues and thereby coordinate physiology and behaviour. We previously showed that the dorsomedial SCN cells run ahead of the remaining cells, and that this differential phasing is an intrinsic property arising from the intercellular network of the SCN. However, the molecular mechanism underlying the phase-advanced oscillations of the dorsomedial cells and how they contribute to behavioural rhythms have remained unknown. In the present study, we have shown that the induction of dorsomedial Per1 expression at dawn is constantly accelerated by the circadian activation of intracellular cAMP signalling, which requires coordinated inhibition of G $\alpha$ i by the clock-controlled RGS16 (see a schematic model in Fig. 5). Importantly, RGS16 deficiency, which delays dorsomedial Per1 expression, results in a period lengthening of behavioural rhythm. These data therefore reveal that the temporal regulation of intracellular $G$ protein signalling that dictates the phasic arrangement within the SCN contributes to the determination of the circadian period of locomotor activity rhythm.

We found that despite the disappearance of the cAMP rhythm, Rgs $16^{-1-}$ mice still show Per1 oscillations in the SCN (Fig. 3). This observation may be somewhat unexpected because pharmacological depletion of cAMP by an irreversible potent AC inhibitor (MDL$12,330 \mathrm{~A})$ constitutively reduces Per1 expression to basal levels ${ }^{29}$. It should be noted, however, that the concentrations of cAMP in the $R g s 16^{-1-}$ SCN (Fig. 2e) and in THFA (a mild AC inhibitor)-treated cells ${ }^{29}$ were not markedly reduced (kept at intermediate levels), and that under these conditions behavioural rhythms were sustainable, albeit with a relatively long circadian period (Figs. 1i and $4 \mathrm{c})^{14}$. Consistent with this behavioural rhythm, the circadian fluctuations of Per1 in the $\mathrm{Rgs}_{16} 6^{-1-} \mathrm{SCN}$ were mostly unimpaired: rhythms in the vast majority of middle-to-ventral cells remained intact, and intriguingly, even in the dorsomedial cells, Per1 was still capable of oscillating, albeit with its peak phase significantly delayed due to RGS16 deficiency (Fig. 3d,e). Thus, the oscillations of Per1 in the SCN are not necessarily depending on the cAMP rhythm, but are rather self-sustainable, presumably due to transcriptional control through E-box and D-box, which might suffice to drive 'normal' (that is, non-phase-leading) Per1 expression. Our data therefore 


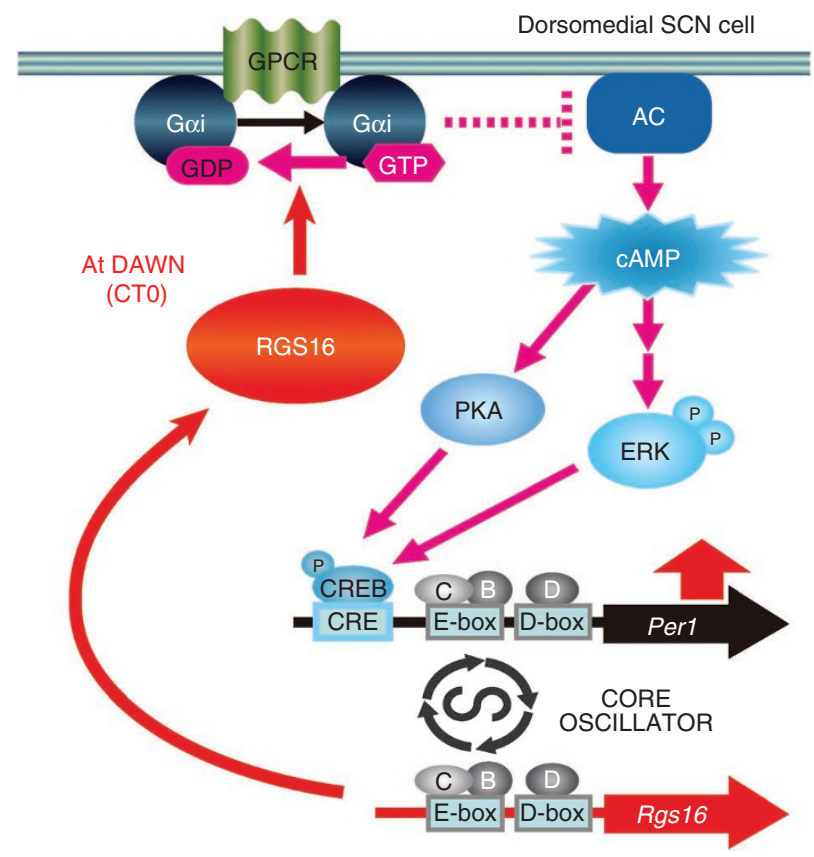

Figure 5 | Schematic model depicting a role of RGS16 in regulated cAMP signalling at dawn in the dorsomedial cells. Through allowing CAMP synthesis at dawn, RGS16 regulates Per1 expression in the dorsomedial phase-leading cells. B, BMAL1; C, CLOCK; CRE, CAMP responsive element; CREB, CRE-binding protein; D, DBP; P, phosphate; ERK, extracellular signalregulated kinase; PKA, CAMP-dependent protein kinase.

propose that the major role of the daily activation of cAMP in the SCN is to accelerate the expression of Per1 in the dorsomedial cells, thereby advancing the phases of these cells, relative to those of the cells in the middle-to-ventral SCN.

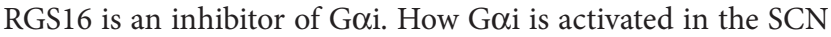
remains unknown. Goi could be activated by extracellular or intracellular signals. One candidate, Dexras1, is a nonreceptor-associated activator for $\mathrm{G \alpha i}^{46}$. Interestingly, the gene encoding Dexras1 is highly and rhythmically expressed in the SCN, with a phase almost anti-phasic to that of Rgs16, and mice lacking Dexras1 show abnormal desynchronization of the behavioural rhythm in constant light ${ }^{47}$. These results raise the prospect that the temporally segregated activation and inhibition of Goi by Dexras1 and RGS16

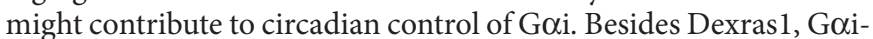
coupled GPCR signalling may also be involved. Defining which specific Goi activators are required for SCN synchrony and how they act in concert with RGS16 will be our next challenge. It is also noteworthy that pharmacological (global) silencing of Goi/o with pertussis toxin treatment could dampen SCN rhythm ${ }^{37}$, a phenotype differing from that of RGS16 deficiency. Thus, the mechanism(s) by which pertussis toxin affects SCN physiology remains unknown. We should also mention that RGS16 can target ${\mathrm{G} \alpha q^{30}}^{30}$. Given that in multiple cell types, Goq-mediated $\mathrm{Ca}^{2+}$ signalling interferes with cAMP production ${ }^{48-50}$, it will be worth testing whether Goq signalling in the SCN is subject to the circadian regulation by RGS16 and how this potential pathway contributes to the altered cAMP signals in the RGS16-deficient SCN.

In line with previous reports, we observed that the expression of $c$ Fos at dawn in the SCN is highly confined to the dorsomedial cells (Fig. 4a) ${ }^{41,42}$. This spatially limited activation of $c$ Fos cannot be explained by RGS16 distribution (Fig. 1c): $c$ Fos expression does not parallel the expression of Rgs16 in middle-to-ventral SCN regions, making it unlikely that RGS16 expression by itself suffices to drive cFos induction. Rather, given that the role of RGS16 is to inhibit
Goi and temporarily permit the production of cAMP in the SCN, it would be reasonable to speculate that RGS16 is necessary (Fig. 4a) but not sufficient to drive cAMP synthesis that triggers the expression of $c F o s$ in the dorsomedial cells. It is conceivable that the circadian regulation of cAMP is a multifactorial process involving not

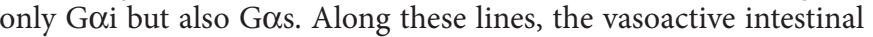
peptide, a neurotransmitter mediating cell-cell communication in the SCN, activates Gos by acting through its cognate receptor $\mathrm{VPAC}_{2}{ }^{8,9,51}$. As it has been speculated that vasoactive intestinal peptide acts on the dorsomedial $\mathrm{SCN}^{52}$, further investigations aimed at deciphering the rules governing the precisely timed and topologically specific activation of Gos and Goi pathways in the SCN will be required to understand how cAMP synthesis in the SCN is controlled.

Because the lengthened period of the $\operatorname{Rgs} 16^{-1-}$ behavioural rhythm (Fig. 1i,j) is closely associated with the delayed phase of the periventricular part of the dorsomedial SCN (Fig. 3a and Supplementary Figs. S7 and S8), we envisage that the functional changes in a small subset of neurons (for example, phase-leading cells) could alter the global timing property of the SCN. We observed that this prediction is mathematically feasible at least in a two-coupled celloscillator model that we constructed in silico (Supplementary Fig. S11). The mathematical configuration used was based on a published model which hypothesizes a mutual coupling between the two oscillators ${ }^{53,54}$ and was modified by introducing a term accounting for the dawn cAMP signal in a phase-leading cell (see Supplementary Methods for details). Under these conditions, the cell oscillating in silico with cAMP signalling at dawn runs ahead of the cell without cAMP signalling, and both cells exhibit the same circadian period (23.6h) (see Supplementary Fig. S11). By contrast, once cAMP signalling was deleted from the phase-leading cell, then the two cells began to oscillate in phase with an elongated circadian period (24.4h) (Supplementary Fig. S11), a situation similar to what was observed for $\mathrm{Rgsl6}^{-/-}$mice under constant dark conditions. Thus, a modified two-coupled cell-oscillator model predicts the period lengthening by delayed circadian oscillation of phase-leading cells, whereas further elucidation of SCN network will be needed to faithfully recapitulate whole SCN function (see Supplementary Methods for detailed discussion).

In conclusion, we provide evidence that RGS16, known as the regulator of intracellular Goi protein signalling, is a key component to establish the intercellular phase-relationship of multi-phased SCN neurons. Mechanistically, RGS16 forms an integration point whereby the intracellular molecular clock exerts its effect on cAMP signalling (Fig. 5). Through allowing cAMP synthesis at dawn, RGS16 in turn facilitates the phase-advanced expression of Per1 in the dorsomedial cells. Thus, our work adds a new layer of complexity to the rules that govern the intercellular synchrony of SCN neurons. We propose that signalling at the cell surface is not everything. Rather, the circadian time-dependent regulation of intracellular $G$ protein signalling is also important to coordinate temporal and spatial order across the SCN. Furthermore, our work extends the role of RGS16 from cellular biology to behaviour. A major challenge in systems neuroscience is to integrate intracellular molecular events with the intercellular neural organization that gives rise to complex behavioural states. Using the SCN as a model, we show that the regulated intracellular G-protein signalling that controls intercellular synchrony across the SCN contributes to the determination of the period of daily rhythms in behaviour.

\section{Methods}

Creation of Rgs16 knockout mice. Rgs16 mutant mice (Acc. No. CDB0436K: http://www.cdb.riken.jp/arg/mutant\%20mice\%20list.html) were generated according to our standard method ${ }^{55}$. The targeting vector was constructed using a bacterial artificial chromosome clone containing the mouse Rgs16 gene (RP24-576N23, BACPAC). We generated the $5^{\prime}$ - and $3^{\prime}$-arms of Rgs 16 (Fig. 1d) by PCR and cloned them into the corresponding sites of the DT-A/lox71/LacZ-pA/frt/PGK-Neo/frt/loxP/pA 
vector (the details of the vector are available in http://www.cdb.riken.go.jp/arg/ cassette.html). Gene targeting was carried out in TT2 cells ${ }^{56}$, an embryonic stem cell line established from F1 embryo between C57BL/6J female and CBA male (50\% C57BL/6J, 50\% CBA). Chimeric males were bred to wild type C57BL/6 females producing F1N0 progeny (75\% C57BL/6J, 25\% CBA). Germline transmission was verified by PCR as well as Southern blotting on tail DNA. F1N0 males were further backcrossed with C57BL/6J females to create F1N1 progeny $(87.5 \%$ C57BL/6J). For behavioural analysis (Fig. 1i,j), we continued back-crossing up to F1N10 generation, which corresponds to 12 times backcrossing to the C57BL/6J background ( $>99.9 \% \mathrm{C} 57 \mathrm{BL} / 6 \mathrm{~J})$ as the F1N0 mice with which we started backcrossing were already 75\% C57BL/6J background. To conduct biochemical and histological analyses, we used F1N5 generation mice ( 99.2\% C57BL/6J), the behavioural phenotypes of which are equivalent to those of F1N10 mice (freerunning period (h) of F1N5 generation mice: $R g s 16^{+/+}, 23.58 \pm 0.03$ (s.e.m.), $R g s 16^{-1-}$, $24.35 \pm 0.03, n=6$ for each genotype, $P<0.01, t$-test). We analysed littermate wild-type control mice having equivalent genetic backgrounds than the mutant mice. To this end, males and females from a specific generation (either F1N5 or F1N10 mice) were interbred to create a large colony. No difference in gross morphology or fertility was detected between the genotypes. Unless otherwise mentioned, in vivo comparative analyses of WT and mutant mice were conducted in DD. All the studies were approved by the animal experimentation committee of Kyoto University.

Behavioural activity monitoring. Single caged adult $R g s 16^{-1-}$ mice and their wild-type littermates (male, 8-15 weeks old) were housed individually in lighttight, ventilated closets within a temperature- and humidity-controlled facility The animals were entrained on a 12-h-light ( 200 lux fluorescent light $)$-12-h-dark cycle at least two weeks and then transferred to DD. Locomotor activity was detected with passive (pyroelectric) infra-red sensors (FA-05 F5B; Omron ${ }^{24,57}$ and the data obtained were analysed with Clocklab software (Actimetrics) developed on MatLab (Mathworks). Free-running period was determined with either a $\chi^{2}$ periodogram or a linear regression line fit to the activity onsets. The data used for period measurements were based on animal behaviours in a 14-day interval taken 3 days after the start of DD condition. For the pulse-induced shift experiments, mice put in DD were exposed to a 15 min light pulse at either CT14 or CT22. Phase shifts (delay at CT14, advance at CT22) were quantified as the time difference between regression lines of activity onset before and after the light application.

Microdissection of the mouse SCN. The microdissection of the SCN was performed as described ${ }^{27}$ with slight modifications. In brief, animals kept in DD were killed by cervical dislocation, and the eyes were removed under a safety red light. The brain was then isolated from the skull under room light and frozen immediately on dry ice. Coronal brain section $(300 \mu \mathrm{m}$ thick) containing the SCN was prepared using a cryostat microtome (CM3050S, Leica) and mounted on a silicon rubber stage at $-17^{\circ} \mathrm{C}$. Under a magnifying glass, the bilateral SCN was punched out from the frozen section using a blunt 20 -gauge syringe needle whose edge had been sharpened by filing. For the analysis of circadian expression of RGS16 and Goi (Fig. 2b), the SCN punches ( $n=3$, for each time point) were pooled in Laemmli buffer, and the lysates were subjected to Western blot analysis with antibodies to RGS16 and Gai. We generated rabbit polyclonal RGS16 antibody. The details of the antibodies used are available in Supplementary Methods.

Pull down assay with His-tagged RGS16. RGS16 binding assay was performed as described ${ }^{58}$ with slight modifications. In brief, the $\mathrm{SCN}$ punches $(n=5)$ were pooled in $300 \mu \mathrm{l}$ of pull-down buffer ( $20 \mathrm{mM}$ Na-HEPES, pH 8.0, $380 \mathrm{mM} \mathrm{NaCl}, 3 \mathrm{mM}$ DTT, $6 \mathrm{mM} \mathrm{MgCl}_{2}$ ) and homogenized by sonication at $4^{\circ} \mathrm{C}$ (Bioruptor, COSMO $\mathrm{BIO})$. The homogenate was divided into two aliquots and incubated with His-RGS16 protein $(10 \mu \mathrm{g})$ in the presence of either GDP $(10 \mu \mathrm{M})$ or GDP $(10 \mu \mathrm{M})$ plus $\mathrm{AlF}_{4}^{-}(30 \mu \mathrm{M})$ for $30 \mathrm{~min}$ at $4^{\circ} \mathrm{C}$. The reaction mixtures were then treated with $1 \%$ cholate and centrifuged at 105,000 $\mathrm{g}$ (Optima MAX ultracentrifuge, Beckman). The detergent-soluble protein extracts were incubated with Ni2 +-NTA agarose beads (Qiagen) for $15 \mathrm{~min}$ at $4{ }^{\circ} \mathrm{C}$. Following three time washes with pull-down buffer supplemented with $20 \mathrm{mM}$ imidazole (plus $10 \mu \mathrm{M}$ GDP with or without $\left.30 \mu \mathrm{M} \mathrm{AlF}_{4}{ }^{-}\right)$, the beads were boiled in Laemmli buffer. The protein complexes copurified with His-tagged RGS16 were analysed by Western blot.

Immunoprecipitation with RGS16 antibody. Immunoprecipitaion (IP) of RGS16 was performed with the SCN protein extracts from $\mathrm{Rgs} 16^{+/+}$and $\mathrm{Rgs} 16^{-/-}$mice. The microdissected SCN punches $(n=5$, for each pool) were homogenized in $300 \mu \mathrm{l}$ of IP buffer (20 mM Na-HEPES, pH 8.0, $380 \mathrm{mM} \mathrm{NaCl}, 3 \mathrm{mM}$ DTT, $6 \mathrm{mM}$ $\mathrm{MgCl}_{2}, 10 \mu \mathrm{M} \mathrm{GDP}$ and $30 \mu \mathrm{M} \mathrm{AlF}_{4}^{-}$) by sonication at $4{ }^{\circ} \mathrm{C}$ (Bioruptor, COSMO $\mathrm{BIO})$. The homogenates were treated with $1 \%$ cholate and then centrifuged at $105,000 \mathrm{~g}$ (Optima MAX, Beckman). The detergent-soluble protein extracts were subjected to IP with RGS16 antibody and Protein A-Sepharose beads. After three time washes with IP buffer, the beads were boiled in Laemmli buffer. The proteins precipitated with RGS16 antibody were analysed by Western blot.

CAMP measurement. cAMP concentration was determined by enzyme immunoassay (EIA) using cAMP EIA kit (Cayman Chemical) according to the manufacturer's protocol. The microdissected SCN (one punch per assay) was collected in $300 \mu \mathrm{l}$ of $0.1 \mathrm{~N} \mathrm{HCl}$ solution and then sonicated at $4^{\circ} \mathrm{C}$ (Bioruptor, COSMO BIO). The protein content was determined with BCA Protein Assay kit (Takara). Following centrifugation at $1,000 \mathrm{~g}$ for $10 \mathrm{~min}$, the supernatant was diluted 1:2 with EIA buffer (Cayman Chemical). All SCN samples underwent acetylation before determination of cAMP level by EIA. We conducted the assays in duplicate, with measurement at $\mathrm{l}=405 \mathrm{~nm}$ on a plate reader (ARVO, Perkin-Elmer).

Per1-Iuc SCN slice culture. Per1-luc transgenic mice carry a firefly luciferase reporter gene linked to a $7.2 \mathrm{~kb}$ genomic DNA fragment covering the $5^{\prime}$ upstream region of the mouse Per1 gene ${ }^{59}$. Perl-luc-Rgs $16^{-1-}$ mice were generated by crossing Per1-luc transgenic mice and Rgs $16^{-1-}$ mice. The SCN slices were prepared according to our standard method ${ }^{6}$ and kept at $35^{\circ} \mathrm{C}$ in a sealed $35 \mathrm{~mm}$ petri dish with $1 \mathrm{ml}$ of the culture medium containing $1 \mathrm{mM}$ luciferin. The bioluminescence from the cultured SCN was measured with a highly sensitive cryogenic CCD camera (Spectra Video SV16KV/CT: Pixelvision) equipped with a microscope (Axiovert 135TV: Carl Zeiss). Observed data of images were filtered through a median filter to eliminate cosmic-ray-induced background noise. The luminescence was recorded every $20 \mathrm{~min}$. Periods of SCN luminescence recordings were estimated using Fast Fourier Transform-Nonlinear Least Squares analysis ${ }^{60}$ as part of the Biological Rhythms Analysis Software System (BRASS). For all SCN to be compared, identical segments of raw SCN luminescence values, starting from the first trough since the start of the measurement, and containing at least four complete cycles, were analysed. BRASS has been developed by Dr Brown PE and co-workers and is available from http://millar.bio.ed.ac.uk/Downloads.html.

Intraventricular infusion of THFA. THFA (Tocris) was continuously delivered to the SCN via osmotic pump as described ${ }^{29}$. In brief, the tip of the infusion cannula (Alzet, Brain Infusion Kit 2) was directed stereotaxically to the SCN and the osmotic pump (Alzet, pump model 1002) containing $100 \mathrm{mM}$ THFA $(118 \mu \mathrm{g} / \mathrm{day}$, infused at $0.24 \mu \mathrm{l} / \mathrm{h})$ was placed in a subcutaneous pouch by surgery under anaesthesia. We performed the surgery during the subjective daytime (CT8-10) when the phase of circadian clock is considered irresponsive to light. Following the surgery, animals were returned to their home cages and maintained in $\mathrm{DD}$.

Statistical analysis. For the experiments in which three or more test groups were compared, we used one-way analysis of variance (ANOVA) with Bonferron post-hoc test and evaluated the differences derived from genotypes and circadian time points tested. To assess the difference of circadian rhythm (phase) between the zones in Per1-luc SCN slices, we analysed the interaction between zone and circadian time with two-way repeated measures ANOVA, followed by Bonferroni's multiple comparison test.

Additional methods. Specific reagents and detailed methodology for DNA microarray analysis, in situ hybridization, X-gal staining, immunoblotting, immunohistochemistry and the antibodies used are all described in Supplementary Methods. Microarray data has been deposited in the Gene Expression Omnibus under accession code GSE28574.

\section{References}

1. Ralph, M. R., Foster, R. G., Davis, F. C. \& Menaker, M. Transplanted suprachiasmatic nucleus determines circadian period. Science 247, 975-978 (1990)

2. Silver, R., LeSauter, J., Tresco, P. A. \& Lehman, M. N. A diffusible coupling signal from the transplanted suprachiasmatic nucleus controlling circadian locomotor rhythms. Nature 382, 810-813 (1996).

3. Sujino, M. et al. Suprachiasmatic nucleus grafts restore circadian behavioural rhythms of genetically arrhythmic mice. Curr. Biol. 13, 664-668 (2003).

4. Welsh, D. K., Logothetis, D. E., Meister, M. \& Reppert, S. M. Individual neurons dissociated from rat suprachiasmatic nucleus express independently phased circadian firing rhythms. Neuron 14, 697-706 (1995).

5. Quintero, J. E., Kuhlman, S. J. \& McMahon, D. G. The biological clock nucleus: a multiphasic oscillator network regulated by light. J. Neurosci. 23, 8070-8076 (2003).

6. Yamaguchi, S. et al. Synchronization of cellular clocks in the suprachiasmatic nucleus. Science 302, 1408-1412 (2003).

7. Liu, A. C. et al. Intercellular coupling confers robustness against mutations in the SCN circadian clock network. Cell 129, 605-616 (2007).

8. Aton, S. J., Colwell, C. S., Harmar, A. J., Waschek, J. \& Herzog, E. D. Vasoactive intestinal polypeptide mediates circadian rhythmicity and synchrony in mammalian clock neurons. Nat. Neurosci. 8, 476-483 (2005).

9. Maywood, E. S. et al. Synchronization and maintenance of timekeeping in suprachiasmatic circadian clock cells by neuropeptidergic signalling. Curr. Biol. 16, 599-605 (2006)

10. Welsh, D. K., Takahashi, J. S. \& Kay, S. A. Suprachiasmatic nucleus: cell autonomy and network properties. Annu. Rev. Physiol. 72, 551-577 (2010).

11. Yan, L. \& Okamura, H. Gradients in the circadian expression of Per1 and Per2 genes in the rat suprachiasmatic nucleus. Eur. J. Neurosci. 15, 1153-1162 (2002). 
12. Hamada, T., Antle, M. C. \& Silver, R. Temporal and spatial expression patterns of canonical clock genes and clock-controlled genes in the suprachiasmatic nucleus. Eur. J. Neurosci. 19, 1741-1748 (2004).

13. Reppert, S. M. \& Weaver, D. R. Coordination of circadian timing in mammals. Nature 418, 935-941 (2002).

14. Hastings, M. H., Maywood, E. S. \& O’Neill, J. S. Cellular circadian pacemaking and the role of cytosolic rhythms. Curr. Biol. 18, R805-R815 (2008).

15. Liu, A. C., Lewis, W. G. \& Kay, S. A. Mammalian circadian signalling networks and therapeutic targets. Nat. Chem. Biol. 3, 630-639 (2007).

16. Yamaguchi, S. et al. Role of DBP in the circadian oscillatory mechanism. Mol. Cell. Biol. 20, 4773-4781 (2000).

17. Mitsui, S., Yamaguchi, S., Matsuo, T., Ishida, Y. \& Okamura, H. Antagonistic role of E4BP4 and PAR proteins in the circadian oscillatory mechanism. Genes Dev. 15, 995-1006 (2001).

18. Doi, M., Okano, T., Yujnovsky, I., Sassone-Corsi, P. \& Fukada, Y. Negative control of circadian clock regulator E4BP4 by casein kinase Iepsilon-mediated phosphorylation. Curr. Biol. 14, 975-980 (2004).

19. Akashi, M., Ichise, T., Mamine, T. \& Takumi, T. Molecular mechanism of cell-autonomous circadian gene expression of Period2, a crucial regulator of the mammalian circadian clock. Mol. Biol. Cell 17, 555-565 (2006).

20. Ohno, T., Onishi, Y. \& Ishida, N. A novel E4BP4 element drives circadian expression of mPeriod2. Nucleic Acids Res. 35, 648-655 (2007).

21. Ueda, H. R. et al. System-level identification of transcriptional circuits underlying mammalian circadian clocks. Nature Genet. 37, 187-192 (2005).

22. Travnickova-Bendova, Z., Cermakian, N., Reppert, S. M. \& Sassone-Corsi, P. Bimodal regulation of mPeriod promoters by CREB-dependent signalling and CLOCK/BMAL1 activity. Proc. Natl Acad. Sci. USA 99, 7728-7733 (2002).

23. Balsalobre, A., Marcacci, L. \& Schibler, U. Multiple signalling pathways elicit circadian gene expression in cultured Rat-1 fibroblasts. Curr. Biol. 10, 1291-1294 (2000).

24. Shigeyoshi, Y. et al. Light-induced resetting of a mammalian circadian clock is associated with rapid induction of the mPer1 transcript. Cell 91, 1043-1053 (1997).

25. Akiyama, M. et al. Inhibition of light- or glutamate-induced mPer1 expression represses the phase shifts into the mouse circadian locomotor and suprachiasmatic firing rhythms. J. Neurosci. 19, 1115-1121 (1999).

26. Field, M. D. et al. Analysis of clock proteins in mouse SCN demonstrates phylogenetic divergence of the circadian clockwork and resetting mechanisms. Neuron 25, 437-447 (2000)

27. Yamazaki, S., Maruyama, M., Cagampang, F. R. \& Inouye, S. T. Circadian fluctuations of cAMP content in the suprachiasmatic nucleus and the anterior hypothalamus of the rat. Brain Res. 651, 329-331 (1994).

28. Obrietan, K., Impey, S., Smith, D., Athos, J. \& Storm, D. R. Circadian regulation of cAMP response element-mediated gene expression in the suprachiasmatic nuclei. J. Biol. Chem. 274, 17748-17756 (1999).

29. O’Neill, J. S., Maywood, E. S., Chesham, J. E., Takahashi, J. S. \& Hastings, M. H. cAMP-dependent signalling as a core component of the mammalian circadian pacemaker. Science 320, 949-953 (2008).

30. Bansal, G., Druey, K. M. \& Xie, Z. R4 RGS proteins: regulation of G-protein signalling and beyond. Pharmacol. Ther. 116, 473-495 (2007).

31. Chen, C., Zheng, B., Han, J. \& Lin, S. C. Characterization of a novel mammalian RGS protein that binds to Galpha proteins and inhibits pheromone signalling in yeast. J. Biol. Chem. 272, 8679-8685 (1997).

32. Beadling, C., Druey, K. M., Richter, G., Kehrl, J. H. \& Smith, K. A. Regulators of $\mathrm{G}$ protein signalling exhibit distinct patterns of gene expression and target $\mathrm{G}$ protein specificity in human lymphocytes. J. Immunol. 162, 2677-2682 (1999).

33. Ueda, H. R. et al. A transcription factor response element for gene expression during circadian night. Nature 418, 534-539 (2002).

34. Panda, S. et al. Coordinated transcription of key pathways in the mouse by the circadian clock. Cell 109, 307-320 (2002)

35. Huang, J. et al. Feeding and fasting controls liver expression of a regulator of $\mathrm{G}$ protein signalling (Rgs16) in periportal hepatocytes. Comp. Hepatol. 5, 8 (2006).

36. Gerstner, J. R., Vander Heyden, W. M., Lavaute, T. M. \& Landry, C. F. Profiles of novel diurnally regulated genes in mouse hypothalamus: expression analysi of the cysteine and histidine-rich domain-containing, zinc-binding protein 1, the fatty acid-binding protein 7 and the GTPase, ras-like family member $11 \mathrm{~b}$. Neuroscience 139, 1435-1448 (2006).

37. Aton, S. J., Huettner, J. E., Straume, M. \& Herzog, E. D. GABA and Gi/o differentially control circadian rhythms and synchrony in clock neurons. Proc. Natl Acad. Sci. USA 103, 19188-19193 (2006).

38. Okamura, H. Suprachiasmatic nucleus clock time in the mammalian circadian system. Cold Spring Harb. Symp. Quant. Biol. 72, 551-556 (2007).

39. Gachon, F., Olela, F. F., Schaad, O., Descombes, P. \& Schibler, U. The circadian PARdomain basic leucine zipper transcription factors DBP, TEF, and HLF modulate basal and inducible xenobiotic detoxification. Cell Metab. 4, 25-36 (2006)

40. Tominaga, K., Inouye, S. I. \& Okamura, H. Organotypic slice culture of the rat suprachiasmatic nucleus: sustenance of cellular architecture and circadian rhythm. Neuroscience 59, 1025-1042 (1994).

41. Sumova, A., Travnickova, Z., Mikkelsen, J. D. \& Illnerova, H. Spontaneous rhythm in c-Fos immunoreactivity in the dorsomedial part of the rat suprachiasmatic nucleus. Brain Res. 801, 254-258 (1998).
42. Schwartz, W. J. et al. Differential regulation of fos family genes in the ventrolateral and dorsomedial subdivisions of the rat suprachiasmatic nucleus. Neuroscience 98, 535-547 (2000).

43. Obrietan, K., Impey, S. \& Storm, D. R. Light and circadian rhythmicity regulate MAP kinase activation in the suprachiasmatic nuclei. Nature Neurosci. 1, 693-700 (1998).

44. Lee, H. S., Nelms, J. L., Nguyen, M., Silver, R. \& Lehman, M. N. The eye is necessary for a circadian rhythm in the suprachiasmatic nucleus. Nature Neurosci. 6, 111-112 (2003).

45. Nakaya, M., Sanada, K. \& Fukada, Y. Spatial and temporal regulation of mitogen-activated protein kinase phosphorylation in the mouse suprachiasmatic nucleus. Biochem. Biophys. Res. Commun. 305, 494-501 (2003).

46. Graham, T. E., Key, T. A., Kilpatrick, K. \& Dorin, R. I. Dexras1/AGS-1, a steroid hormone-induced guanosine triphosphate-binding protein, inhibits $3^{\prime}, 5^{\prime}$-cyclic adenosine monophosphate-stimulated secretion in AtT-20 corticotroph cells. Endocrinology 142, 2631-2640 (2001).

47. Cheng, H. Y. et al. Dexras1 potentiates photic and suppresses nonphotic responses of the circadian clock. Neuron 43, 715-728 (2004).

48. von Hayn, K. et al. Gq-mediated $\mathrm{Ca}^{2+}$ signals inhibit adenylyl cyclases $5 / 6$ in vascular smooth muscle cells. Am. J. Physiol. 298, C324-332.

49. Yoshimura, M. \& Cooper, D. M. Cloning and expression of a $\mathrm{Ca}(2+)$ inhibitable adenylyl cyclase from NCB-20 cells. Proc. Natl Acad. Sci. USA 89, 6716-6720 (1992).

50. Chabardes, D., Imbert-Teboul, M. \& Elalouf, J. M. Functional properties of $\mathrm{Ca}^{2+}$-inhibitable type 5 and type 6 adenylyl cyclases and role of $\mathrm{Ca}^{2+}$ increase in the inhibition of intracellular cAMP content. Cell. Signal. 11, 651-663 (1999).

51. Harmar, A. J. et al. The VPAC(2) receptor is essential for circadian function in the mouse suprachiasmatic nuclei. Cell 109, 497-508 (2002).

52. Aton, S. J. \& Herzog, E. D. Come together, right.now: synchronization of rhythms in a mammalian circadian clock. Neuron 48, 531-534 (2005).

53. Lema, M. A., Golombek, D. A. \& Echave, J. Delay model of the circadian pacemaker. J. Theor. Biol. 204, 565-573 (2000).

54. Locke, J. C., Westermark, P. O., Kramer, A. \& Herzel, H. Global parameter search reveals design principles of the mammalian circadian clock. BMC Syst. Biol. 2, 22 (2008).

55. Murata, T. et al. ang is a novel gene expressed in early neuroectoderm, but its nul mutant exhibits no obvious phenotype. Gene Expr. Patterns 5, 171-178 (2004)

56. Yagi, T. et al. A novel ES cell line, TT2, with high germline-differentiating potency. Anal. Biochem. 214, 70-76 (1993).

57. Masubuchi, S., Kataoka, N., Sassone-Corsi, P. \& Okamura, H. Mouse Period1 (mPER1) acts as a circadian adaptor to entrain the oscillator to environmental light/dark cycles by regulating mPER2 protein. J. Neurosci. 25, 4719-4724 (2005).

58. Saitoh, O., Kubo, Y., Miyatani, Y., Asano, T. \& Nakata, H. RGS8 accelerates G-protein-mediated modulation of K+ currents. Nature 390, 525-529 (1997).

59. Yamaguchi, S. et al. The $5^{\prime}$ upstream region of mPerl gene contains two promoters and is responsible for circadian oscillation. Curr. Biol. 10, 873-876 (2000).

60. Plautz, J. D. et al. Quantitative analysis of Drosophila period gene transcription in living animals. J. Biol. Rhythms 12, 204-217 (1997).

\section{Acknowledgments}

We thank M. Jounouchi for their expert assistance. This work was supported by Special Coordination Funds for Promoting Science and Technology from the Ministry of Education, Culture, Sports, Science and Technology, the Japanese Government.

\section{Author contributions}

M.D. and A.I. are equally contributing first authors. H.O. conceived the project. M.D. and H.O. designed the research. A.I., A.M., M.D. R.T., K.N., S.A. and H.O. created Rgs 16 mutant mice. M.D., A.I., A.M. M.S., R.K., F.Y., I.K., S.T., K.S., Y.Y., M.M., Y.S., H.Y., Y.T., K.O., G.T. and H.O. conducted biochemical and histochemical analyses. M.S., K.S. M.M., J.-M.F. M.K. and H.O. conducted slice culture analyses. H.K., M.A. and M.D. performed mathematical modelling. M.D., K.O., J.-M.F. and H.O. drafted the manuscript.

\section{Additional information}

Accession codes: Microarray data has been deposited in the Gene Expression Omnibus under accession code GSE28574.

Supplementary Information accompanies this paper at http://www.nature.com/ naturecommunications

Competing financial interests: The authors declare no competing financial interests.

Reprints and permission information is available online at http://npg.nature.com/ reprintsandpermissions/

How to cite this article: Doi, M. et al. Circadian regulation of intracellular G-protein signalling mediates intercellular synchrony and rhythmicity in the suprachiasmatic nucleus. Nat. Commun. 2:327 doi: 10.1038/ncomms1316 (2011).

License: This work is licensed under a Creative Commons Attribution-NonCommercialShare Alike 3.0 Unported License. To view a copy of this license, visit http:// creativecommons.org/licenses/by-nc-sa/3.0/ 